\title{
Four fermion condensates in $S U(2)$ Yang-Mills-Higgs theory on a lattice
}

\author{
Nouman Butt* and Simon Catterall ${ }^{\dagger}$ \\ Department of Physics, Syracuse University, Syracuse, New York 13244, United States \\ E-mail: ntbuttesyr.edu
}

\begin{abstract}
We study a model of four reduced staggered fields transforming in the bifundamental representation of a $S U(2) \times S U(2)$ symmetry group where just one of the $S U(2)$ factors is gauged. This field content and symmetries are similar to a Higgs-Yukawa model that has been studied recently. The key observation in the latter work is that fermions acquire masses at strong coupling via the formation of a symmetric four fermion condensate in contrast to the more usual symmetry breaking bilinear condensate seen in eg. NJL models. The current work attempts to see whether this structure survives when the four fermi interactions are replaced by gauge interactions and to explore the resulting phase diagram.
\end{abstract}

The 36th Annual International Symposium on Lattice Field Theory - LATTICE2018

22-28 July, 2018

Michigan State University, East Lansing, Michigan, USA.

\footnotetext{
*Speaker.

${ }^{\dagger}$ smcatterall@gmail.com
} 


\section{Introduction}

The motivation to study this particular lattice model stems from previous studies $[3,8,5,10$, 11] of an $S U$ (4) invariant four-fermion model in three dimensions which possesses an unusual phase structure in which fermions acquire masses at strong coupling without forming a symmetry breaking bilinear condensate. A single continuous phase transition with non mean field exponents separates this strong coupling phase from a massless free fermion phase. In four dimensions it appears that a narrow broken phase separates the massless and massive phases [6] but recent work [1] provided evidence that this broken phase can be evaded in a Higgs-Yukawa generalization of the model in which the auxiliary field used to generate the four fermion interaction is given a kinetic term. In this expanded phase diagram there is evidence that once again a direct transition between massless and massive phase is possible. Since there is no symmetry breaking this novel transition eludes a Landau-Ginzburg description in terms of a local order parameter. Instead the transition is argued to result from a proliferation of topological defects in the scalar field [4].

The phenomenon of symmetric mass generation has also received a great deal of interest in condensed matter physics $[12,9,13]$ and in three dimensions this phenomenon was conjectured to be described by a gauge theory [2].

The Higgs-Yukawa models that have been used to generate this novel structure are invariant under an $S O(4)$ symmetry and utilize a scalar field which transforms in the adjoint representation of one of the $S U$ (2) factors making up $S O(4)=S U(2) \times S U(2)$. In the rest of this paper we call this $S U_{+}(2)$. This scalar is a singlet under the other factor which we call $S U_{-}(2)$. It is then rather natural to imagine replacing the adjoint scalar field with a corresponding gauge field and ask whether the resulting gauge theory is capable of generating a four fermion condensate even in the absence of a Yukawa coupling. In practice we have considered a model containing both scalars and gauge fields and tried to map out the resulting phase diagram.

We expect for small Yukawa coupling that the theory is in a confined phase while for weak gauge coupling and large Yukawa coupling one might expect to see the four fermion condensate which can be interpreted as a Higgs phase of the theory. it is plausible that one might expect to see a line of phase transitions separating these two phases the two dimensional phase diagram spanned by the Yukawa and gauge couplings.

\section{Fermion kinetic term}

Consider staggered fermions in the bifundamental representation of an $S U(2) \times S U(2)$ symmetry. The fermions transform under a general gauge transformation as

$$
\psi \rightarrow G \psi H^{\dagger}
$$

where $G \in S U_{+}(2)$ and $H \in S U_{-}(2)$. The above transformation with left and right action of the group is equivalent to the standard tensor transformation

$$
\psi^{A a}=G^{A B} H^{* a b} \psi^{B b}
$$


To construct the model we start with the full staggered action given in (2.3) gauged under both $S U(2)$ factors:

$$
S_{F}=\sum_{x, \mu} \frac{1}{2} \eta_{\mu}(x) \operatorname{Tr}\left[\psi^{\dagger}(x) U_{\mu}(x) \psi(x+\mu) V_{\mu}^{\dagger}(x)-\psi^{\dagger}(x) U_{\mu}^{\dagger}(x-\mu) \psi(x-\mu) V_{\mu}(x-\mu)\right]
$$

This action is invariant under the following gauge transformations

$$
\begin{array}{r}
\psi(x) \rightarrow G(x) \psi(x) H^{\dagger}(x) \\
U_{\mu}(x) \rightarrow G(x) U_{\mu}(x) G^{\dagger}(x+\mu) \\
V_{\mu}(x) \rightarrow H(x+\mu) V_{\mu}(x) H^{\dagger}(x)
\end{array}
$$

The only single site gauge invariant mass term for fields in the bifundamental representation is $\operatorname{Tr}\left(\psi^{\dagger} \psi\right)$ which vanishes on account of the Grassmann nature of the fields. We can, however, construct a gauge invariant four-fermion term given in (2.5)

$$
\operatorname{Tr}\left(\psi^{\dagger} \psi \psi^{\dagger} \psi\right)
$$

which is non-zero and yields the usual four fermion term.

\section{Imposing the reality condition}

The equivalence between this model and the original four fermi models requires the imposition of two further constraints. First we need to impose a reality condition on the fermions to reduce to four real degrees of freedom and second we will eventually set the gauge coupling for $S U_{-}(2)$ to zero and set $V_{\mu}(x)=I$. For the moment tet us focus the first of these which is equivalent to imposing the constraint

$$
\psi^{\dagger}=\sigma_{2} \psi^{T} \sigma_{2}
$$

This implies that the fermion field can be written in terms of four real components $\chi_{\mu}, \mu=1 \ldots 4$

$$
\psi=\sum_{\mu} \chi_{\mu} \sigma_{\mu}
$$

where $\sigma_{\mu}=\left(I, i \sigma_{i}\right)$ and the original $S O(4)$ fields can be recovered using the relation

$$
\chi_{\mu}=\frac{1}{2} \operatorname{Tr}\left(\sigma_{\mu} \psi\right)
$$

Notice that the previous four fermion term $\operatorname{Tr}\left(\psi^{\dagger} \psi \psi^{\dagger} \psi\right)$ reduces to the simple form $\chi_{1} \chi_{2} \chi_{3} \chi_{4}$ after this. Making use of this condition we can write the action as

$$
S_{F}=\sum_{x, \mu} \frac{1}{2} \eta_{\mu}(x) \operatorname{Tr}\left[\psi^{T}(x) \mathscr{U}_{\mu}(x) \psi(x+\mu) \mathscr{V}_{\mu}^{T}(x)-\psi^{T}(x) \mathscr{U}_{\mu}^{T}(x-\mu) \psi(x-\mu) \mathscr{V}_{\mu}(x-\mu)\right]
$$

where

$$
\begin{gathered}
\mathscr{U}_{\mu}(x)=\sigma_{2} U_{\mu}(x) \\
\mathscr{V}_{\mu}(x)=-V_{\mu}(x) \sigma_{2}
\end{gathered}
$$


This fermion operator is manifestly anti-symmetric

$$
M=\sum_{\mu} \mathscr{U}_{\mu}(x) \delta(x+\mu, x) \mathscr{V}_{\mu}^{T}(x)-\mathscr{U}_{\mu}^{T}(x-\mu) \delta(x-\mu, x) \mathscr{V}_{\mu}(x-\mu)
$$

where $\mathscr{V}$ acts from the right. This form of the action leads to a Pfaffian rather than a determinant after the fermion integration. Moreover this fermion operator inherits the reality condition

$$
M^{*}=\sigma_{2} M \sigma_{2}
$$

Combining anti-symmetry and pseudo-reality we expect $M$ to exhibit a quartet of complex eigenvalues $(\lambda, \bar{\lambda},-\lambda,-\bar{\lambda})$. This guarantees that the fermion operator will have generically possess a real, positive definite Pfaffian. The exception to this will be if the operator develops a purely real eigenvalue. We have not observed this to be the case in our work.

In fact the situation is even better than this. Let us now return to the second constraint on the model. The model we are finally interested has only one set of gauge fields corresponding to $S U_{+}(2)$. If the gauge links corresponding to $S U_{-}(2)\left(V_{\mu}(x)=\mathbb{1}\right)$ are set to unity all the eigenvalues of $M$ are doubled and positivity is then completely guaranteed.

\section{Adding Yukawa interactions}

To facilitate the formation of a gauge invariant four fermion interaction we add the term given in (2.5) after which the action is

$$
\begin{aligned}
S_{F}= & \frac{1}{2} \sum_{x, \mu} \eta_{\mu}(x) \operatorname{Tr}\left[\psi^{T}(x) \mathscr{U}_{\mu}(x) \psi(x+\mu) \sigma_{2}\right]-\frac{1}{2} \sum_{x, \mu} \eta_{\mu}(x) \operatorname{Tr}\left[\psi^{T}(x) \mathscr{U}_{\mu}^{T}(x-\mu) \psi(x-\mu) \sigma_{2}\right] \\
& +\frac{G^{2}}{4} \sum_{x} \operatorname{Tr}\left(\psi^{T} \sigma_{2} \psi \sigma_{2} \psi^{T} \sigma_{2} \psi \sigma_{2}\right)
\end{aligned}
$$

As usual an action quadratic in fermionic variables can be achieved if we introduce an auxiliary field. In this case there are two fermion bilinears defined in (4.2) each of which transforms in the adjoint representation under one of the $S U(2)$ 's and is a singlet under the other. Here we have used $\psi^{\dagger}$ rather than $\psi^{T}$ to exhibit more clearly the transformation properties of each bilinear.

$$
\begin{aligned}
& \psi^{\dagger} \psi \rightarrow H \psi^{\dagger} \psi H^{\dagger} \\
& \psi \psi^{\dagger} \rightarrow G \psi \psi^{\dagger} G^{\dagger}
\end{aligned}
$$

The two possible auxiliary fields $\phi(x)$ and $\sigma(x)$ must transform as

$$
\begin{gathered}
H \sigma H^{\dagger} \\
G \phi G^{\dagger}
\end{gathered}
$$

Since in the end we will choose to gauge only $S U_{+}(2)$ we choose to include only the $\phi$ auxiliary field in our work.

$$
\begin{aligned}
S_{F}= & \frac{1}{2} \sum_{x, \mu} \eta_{\mu}(x) \operatorname{Tr}\left[\psi^{T}(x) \mathscr{U}_{\mu}(x) \psi(x+\mu) \sigma_{2}\right]-\frac{1}{2} \sum_{x, \mu} \eta_{\mu}(x) \operatorname{Tr}\left[\psi^{T}(x) \mathscr{U}_{\mu}^{T}(x-\mu) \psi(x-\mu) \sigma_{2}\right] \\
& +\frac{G}{2} \sum_{x} \operatorname{Tr}\left[\psi^{T}(x) \sigma_{2} \phi(x) \psi(x) \sigma_{2}\right]+\frac{1}{2} \sum_{x} \operatorname{Tr}\left[\phi^{2}\right]
\end{aligned}
$$


Notice that the field $\phi$ is strictly forbidden from picking up a vev as that would imply spontaneous breaking of gauge symmetry which is forbidden in a lattice gauge theory ${ }^{1}$. To test for spontaneous breaking of the global $S U_{-}(2)$ symmetry we can also add a gauge invariant mass term given in (4.5) to the action

$$
m \sum_{x} \operatorname{Tr}\left[\sigma_{3} \psi^{\dagger} \psi\right]
$$

This term explicitly breaks $S U_{-}(2) \rightarrow U(1)$. Finally, for the gauge part of action we have employed the standard Wilson action

$$
S_{G}=\sum_{x} \sum_{\mu<v}-\frac{\beta}{2 N} \operatorname{Tr}\left[U_{\mu v}(x)+U_{\mu v}^{\dagger}(x)\right]
$$

\section{Reduction to $S O(4)$ model}

To connect this model to the original $S O(4)$ model studied in [3] we consider the limit $\beta \rightarrow \infty$ which allows us to set $U_{\mu}(x)=\mathbb{1}$. The fermion operator then reduces to a symmetric difference operator and the action becomes

$$
S=\sum_{x, \mu} \frac{1}{2} \operatorname{Tr}\left[\psi^{T} \sigma_{2}(\eta . \Delta) \psi \sigma_{2}\right]+\frac{G}{2} \sum_{x} \operatorname{Tr}\left[\psi^{T}(x) \sigma_{2} \phi(x) \psi(x) \sigma_{2}\right]+\frac{1}{2} \sum_{x} \operatorname{Tr}\left[\phi^{2}(x)\right]
$$

Transforming in the fundamental of $S O(4)$ one finds

$$
\begin{aligned}
S= & \sum_{x, \mu} \frac{1}{2} \chi^{a}(\eta . \Delta) \delta^{a b} \chi^{b} \\
& +\frac{G}{2} \sum_{x}\left[\phi_{1}\left(\chi_{1} \chi_{2}+\chi_{3} \chi_{4}\right)+\phi_{2}\left(\chi_{1} \chi_{3}+\chi_{2} \chi_{4}\right)+\phi_{3}\left(\chi_{1} \chi_{4}+\chi_{2} \chi_{3}\right)\right]+\frac{1}{2} \sum_{x}\left(\phi_{i}\right)^{2}
\end{aligned}
$$

Notice that the fermion bilinears appear in the self-dual representation of $S O(4)$ in these variables. This projection on self-dual fields can then be transferred to the auxiliary field and after performing the trace over $S U(2)$ indices we recover the $S O(4)$ invariant action studied in [3]

$$
S=\sum_{x, \mu} \frac{1}{2} \chi^{a}\left[(\eta \cdot \Delta) \delta^{a b}+\frac{G}{2} \phi_{+}^{a b}\right] \chi^{b}+\frac{1}{2} \sum_{x}\left(\phi_{+}^{a b}\right)^{2}
$$

\section{Numerical Results}

Now we come to some preliminary results obtained by using the RHMC algorithm to simulate the model in (4.4). Our code utilizes the MILC libraries to allow for efficient parallelization to allow for studies on large lattice. However, our results so far have been confined to a small volume $4^{4}$ lattice suitable for testing and validation of the code.

One important test of our code is whether we recover the known behavior of the $S O(4)$ model in the weak gauge coupling limit. For this purpose we switch off the gauge fields and scan the four fermion condensate as a function of G.This is plotted in Fig. 1(left). Another proxy observable for the four fermion (massive) phase is $\operatorname{Tr}\left(\phi^{2}\right)$ which is plotted in Fig. 1(right). This plots are 

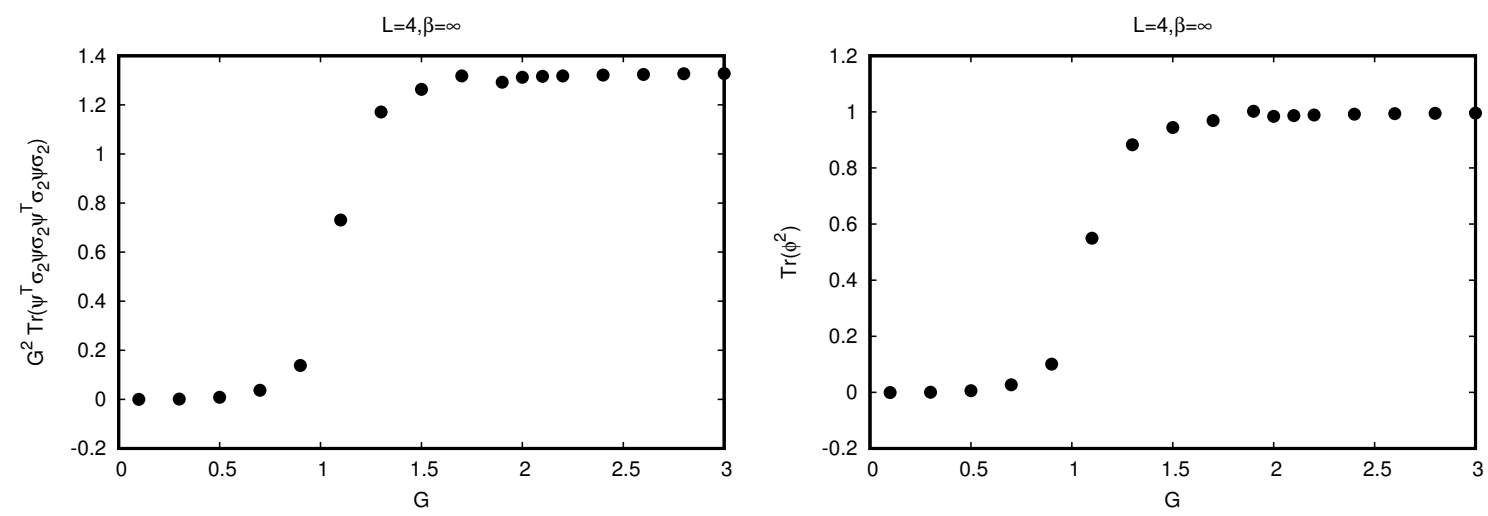

Figure 1: Four-fermion condensate(left) and $\operatorname{Tr}\left(\phi^{2}\right)$ (right) vs $G$ with $\beta=\infty$ for $L=4$
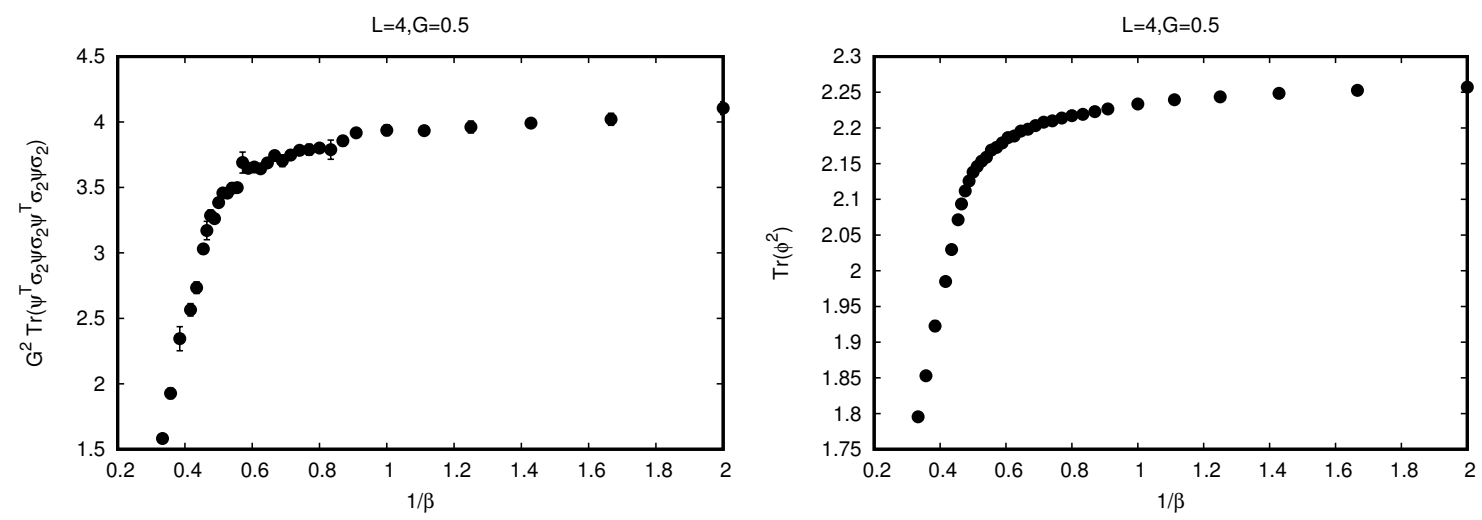

Figure 2: Four-fermion condensate(left) and $\operatorname{Tr}\left(\phi^{2}\right)$ (right) vs $1 / \beta$ with $G=0.5$ for $L=4$

consistent with those reported in [3]. Of course our main goal is to try to realize a four fermion phase through gauge interactions. As a first step in this direction we plot in Fig. 2 the four fermion condensate and $\left\langle\operatorname{Tr}\left(\phi^{2}\right)\right\rangle$ vs $1 / \beta$ at $G=0.5$. At strong gauge coupling the four fermion condensate rises rapidly to a non-zero constant value. Notice that this value of $G$ would not be sufficient to realize a four fermion phase in the absence of gauge interactions. It remains to map out this transition line for a range of $G$ (using much larger lattice volumes).

\section{Summary}

In this paper we have embedded an $S O(4)$ invariant four-fermion model into a gauge model by gauging one the two $S U(2)$ subgroups of $S O(4)$. We have described in some detail the construction of the model in terms of a staggered fermion field transforming in the bifundamental representation of $S U(2) \times S U(2)$ and how this representation can be related to the original fundamental representation of $S O(4)$ by imposing a suitable reality condition on the fermions. Our numerical work is only just beginning but confirms that the model does indeed reduce to the original four fermion

\footnotetext{
${ }^{1}$ Unfortunately while $\phi$ is a singlet Under $S U_{-}(2)$ it does not obey the reality condition so that octct structure of eigenvalues of the fermion operator is reduced once again to quartets.
} 
model in the limit the gauge coupling is sent to zero. Once the gauge coupling is turned on we have so far only run with a single value of the Yukawa coupling on a small lattice but our results are compatible with the appearance of a four fermion phase driven in part by strong gauge interactions. We interpret the four fermion phase as the (gauge invariant) signal of a Higgs phase in the gauge theory.

Future work will aim to map out the phase diagram of the model using much larger lattices and understand the nature of any critical lines encountered. It will be particularly interesting to see whether the four fermion phase survives the limit in which the Yukawa coupling is sent to zero.

\section{References}

[1] N. Butt, S. Catterall and D. Schaich, arXiv:1810.06117 [hep-lat].

[2] Y. Z. You, Y. C. He, C. Xu and A. Vishwanath, Phys. Rev. X 8, no. 1, 011026 (2018)

[3] S. Catterall and D. Schaich, Phys. Rev. D 96, no. 3, 034506 (2017)

[4] S. Catterall and N. Butt, Phys. Rev. D 97, no. 9, 094502 (2018)

[5] S. Catterall, JHEP 1601, 121 (2016)

[6] D. Schaich and S. Catterall, EPJ Web Conf. 175, 03004 (2018) doi:10.1051/epjconf/201817503004

[7] Y. Z. You, Y. C. He, A. Vishwanath and C. Xu, Phys. Rev. B 97, no. 12, 125112 (2018)

[8] V. Ayyar and S. Chandrasekharan, JHEP 1610, 058 (2016)

[9] T. Morimoto, A. Furusaki and C. Mudry, Phys. Rev. B 92, no. 12, 125104 (2015)

[10] V. Ayyar and S. Chandrasekharan, Phys. Rev. D 91, no. 6, 065035 (2015)

[11] V. Ayyar and S. Chandrasekharan, Phys. Rev. D 93, no. 8, 081701 (2016)

[12] K. Slagle, Y. Z. You and C. Xu, Phys. Rev. B 91, no. 11, 115121 (2015)

[13] Y. Y. He, H. Q. Wu, Y. Z. You, C. Xu, Z. Y. Meng and Z. Y. Lu, Phys. Rev. B 94, no. 24, 241111 (2016) 